\title{
Calibration and monitoring of the ATLAS Tile calorimeter
}

\author{
J. Carvalho \\ LIP-Coimbra, Departamento de Física, Universidade de Coimbra, 3004-516 Coimbra, Portugal \\ E-mail: jcarlos@lipc.fis.uc.pt \\ (for the ATLAS Tilecal Collaboration)
}

\begin{abstract}
.
The calibration and monitoring systems of the ATLAS hadronic calorimeter, the TileCal, are presented. Special attention is given to the experience gained so far and perspectives for the future, alongside with the results obtained from the analysis of data acquired during the testbeam periods.
\end{abstract}

Keywords: hadron calorimetry, calibration, monitoring

PACS: PACS numbers: 07.20.Fw, 06.20.Fn

\section{INTRODUCTION}

The ATLAS hadronic sampling calorimeter is built from iron absorbers and scintillator tiles. It is divided into three parts along its axis, a central and two extended cylindrical barrels, each part divided in 64 wedges. The TileCal covers a pseudorapidity up to $|\eta|=1.7$. The good hermeticity is $\phi$ was obtained using a vertical placement of the scintillating tiles in the $r-\phi$ plane. Each tile is readout by two wavelength shifting fibers, coupled to photomultipliers. The fibers are joined in bundles in such a way to form projective towers pointing to the interaction region, to reach the desired segmentation, divided in three longitudinal samples (with different interaction lengths), with a granularity of $\eta * \phi=0.1 * 0.1$ [1].

Several systems are used to calibrate and monitor the calorimeter performance. The charge injection system is used to calibrate the relative response of the photomultiplier readout electronics, across all the calorimeter, and to track any variation with time. The laser system is designed to calibrate and monitor the response of the photomultipliers, in particular the stability of their gains, their global linearity, and for timing studies. A cesium radioactive source system is designed to measure the quality of the optical response of each calorimeter cell, to equalize the signal response from all the cells and to monitor it with time. Finally the minimum bias current will be used to continuously monitor the calorimeter response during physics runs. About $12 \%$ of the modules were calibrated in a testbeam with electrons, muons and pions [2].

\section{CALIBRATION AND MONITORING OF THE TILECAL}

Each TileCal cell can be divided, for monitoring and calibration purposes, into three parts: the optical part (the scintillating tiles where the light is produced by the charged 


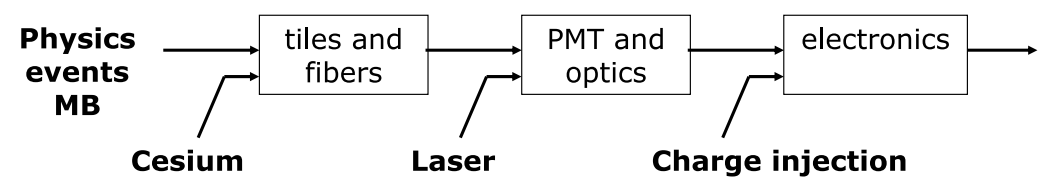

FIGURE 1. Diagram of the optical and electronic readout, and the calibration and monitoring systems.

particles and the wavelength shifting fibers that transport the light into the photomultipliers); the photomultiplier tube (PMT) that converts and amplifies the optical signal; and the readout electronics that shapes, amplify and digitizes the signal from the PMTs [3]. For each one of these parts a dedicated monitoring and calibration system was designed and built, which are described in the next sections. Figure 1 shows a diagram of the sequence of the TileCal optical and electronic readout, and the location of the calibration and monitoring systems inputs.

\subsection{Charge injection system}

The charge injection system (CIS) is designed to calibrate the relative response of the pulse readout electronics for all calorimeter PMTs and to monitor the variations with time. The objective is an accuracy of $1 \%$. Each PMT channel has two analogue paths (bi-gain), the high and low gain ( 82 counts/pC and 1.3 counts/pC, respectively), digitized by a 10-bit ADC, covering a range of $800 \mathrm{pC}$ (corresponding to a energy deposition of about $700 \mathrm{GeV}$ ). The CIS constants allows for the conversion of ADC counts to energy (in units of $\mathrm{pC}$ ).

Each channel is equipped with calibration capacitors, charged from a high precision voltage source and discharged into the input electronics. The resultant waveform is similar to the one produced by the PMT for a given charge, except for an amplitude $10 \%$ larger and a FWHM 10\% smaller. Dedicated CIS runs are taken, scanning the full range of charges for both gains, allowing for the determination of the ADC counts/pC ratio for each channel. The pulse amplitude is reconstructed, from ADC samples taken every $25 \mathrm{~ns}$, with a 3-parameter fit. An example of this fit is shown in fig. 2.

The response as a function of the injected charge is shown in fig. 3 over the full range of both gains. Both the total response and the residuals from a linear fit are shown, and the deviation from linearity is no more than 2.2 counts over the active range of both gains.

The channel-to-channel variation on the measured gains has a RMS of $1.5 \%$ before applying any correction, comparable with the $1 \%$ variation in the calibration capacitors. During a 4-month period the corrected gains were stable at a RMS level better than $0.2 \%$.

In ATLAS there will be periodic CIS runs over the full dynamic range during beamoff periods (between LHC fills and maintenance periods). Interleaved with data are also mono-CIS events, where a fixed amplitude signal is injected during missing bunches in 


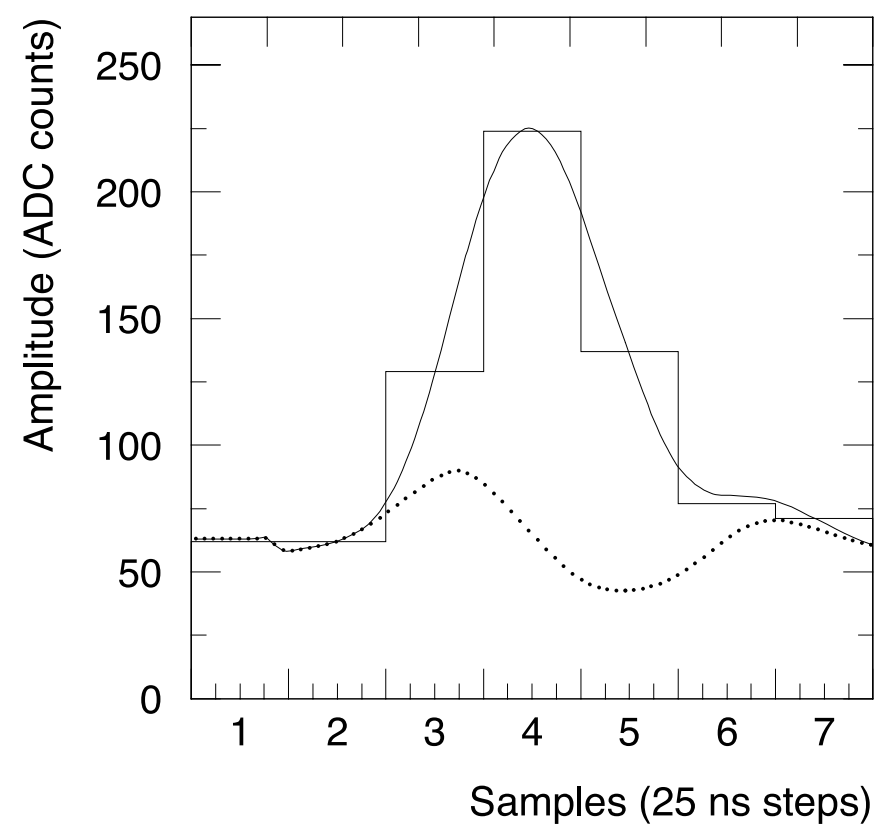

FIGURE 2. CIS pulse of a given channel. The histogram shows the ADC samples taken in 25 ns steps and the solid line corresponds to the 3-parameter fit. The contribution of the switch capacitance is shown as the dotted curve.

the LHC beam structure.

\subsection{Laser system}

The laser system was designed to calibrate and monitor the PMTs response with an accuracy better that $0.5 \%$. It is used to check the PMTs linearity, for studies on pulse saturation recovery and in calorimeter timing, and also for debugging and diagnostics tests.

A frequency doubled Nd:YVO4 laser is used to produce pulses with a wavelength of $532 \mathrm{~nm}$ and $\mathrm{a} \sim 10 \mathrm{~ns}$ width, synchronized with the bunch-crossing clock. Close to the laser the light is split, with part of it being sent to a set of photodiodes for relative intensity monitoring, and the remaining being delivered, through a light distribution system and clear optical fibers, to each calorimeter PMT.

The short and long term monitoring of the PMTs gain stability is the most important role of the system. This is done in special calibration runs from the ratio of the charge measured by each PMT, for a given laser pulse, to the photodiodes response. This ratio provides a reference for the relative gain of each PMT. The results obtained in testbeam conditions show that the PMTs are stable and that the laser system accuracy matches the design goal.

The linearity of the PMTs can be studied varying, in a known range, the intensity of the light delivered. Although the laser intensity itself can be varied only by a factor 10 , 

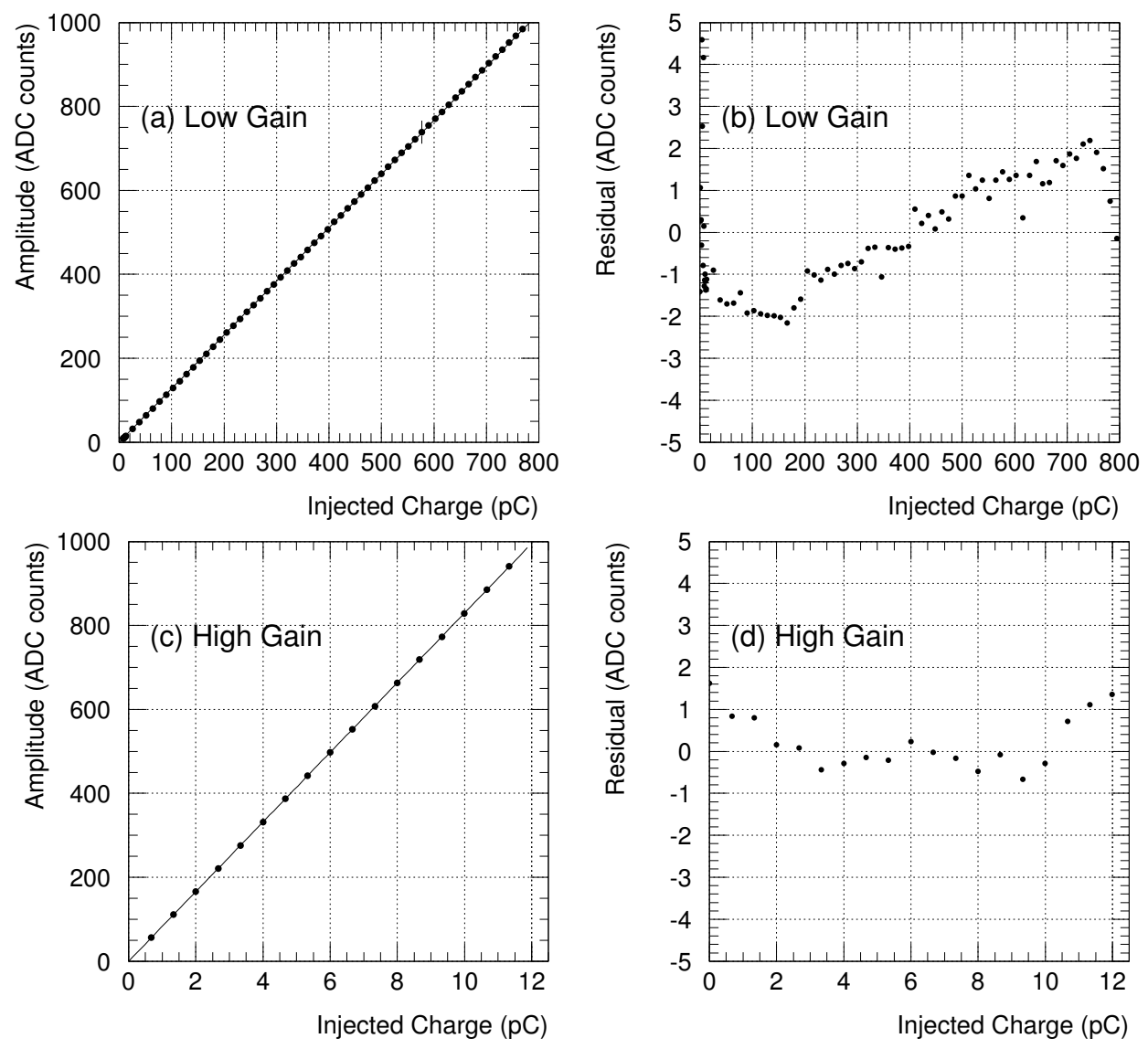

FIGURE 3. Amplitude of the response of the low gain, top, and high gain, bottom, paths of a single channel as a function of the injected charge. The right plots show the residuals from a linear fit.

an automated set of filters allows an extra dynamic range of over 1000. The global PMTs non-linearity was found to be better than $1 \%$ in the $0.7-5 \mathrm{pC}$ range, $0.7 \%$ in the $5-80 \mathrm{pC}$ range and $0.3 \%$ in the $80-700 \mathrm{pC}$ range.

The laser system is also used to measure the absolute gain of each PMT, and the photoelectron yield can be obtained from the combination with the measured calorimeter response in $\mathrm{pC} / \mathrm{GeV}$. It takes advantage of the fact that the pulse-to-pulse intensity fluctuations of the laser are accurately tracked by the monitoring photodiodes. The laser system is also useful in timing studies, as taking into account the differences in propagation of signals, the timings done with projective particles and with laser pulses can be easily correlated, as shown in fig. 4. High intensity laser pulses can be sent to the PMTs in order to saturate the readout response and then apply saturation recovery strategies.

In ATLAS there will be special multi-pulse laser runs, linearity runs over the full dynamics $(\sim 60000)$, but also mono-pulse events inside empty bunches. In addition there will be saturation studies (with pulses well above the $800 \mathrm{pC}$ limit $(\sim 1.4 \mathrm{TeV} /$ cell)). 


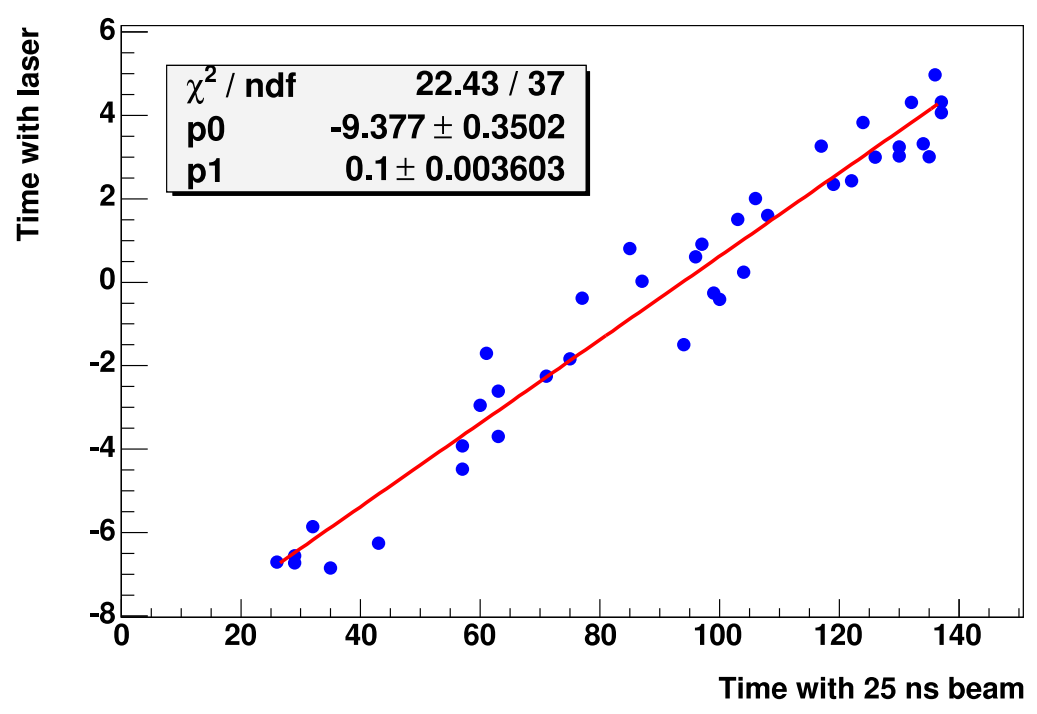

FIGURE 4. The timing with the laser system as a function of the timing with the $25 \mathrm{~ns}$ beam is shown.

\subsection{Cesium source system}

The cesium calibration is designed to determine the quality of the optical response of each calorimeter cell, to adjust the PMTs high voltage in order to equalize the response from all cells and to monitor it with time [4]. The objective is to keep the stability of the energy calibration at the $0.5 \%$ level. The system uses a ${ }^{137} \mathrm{Cs} \gamma$ source which moves, orthogonally to the tile planes, through a hole in the scintillating tiles. This source is transported by a hydraulic system in a series of straight paths along the calorimeter modules, so it passes through holes in every single scintillating tile and absorber plate. The current from each PMT is measured with an integrator which samples the signal at a $90 \mathrm{~Hz}$ rate [5]. The response of the individual tiles is clearly seen as the mean path of the $\gamma_{\mathrm{s}}$ is comparable with the periodic $18 \mathrm{~mm}$ separation between tiles. Figure 5 shows the scan of three adjacent cells where the response of the individual tiles is clearly seen and the effect of a poor optical coupling of a tile to the fiber is evident.

Two methods are used to measure the calorimeter response. In the first of them, the "integral method", the area of the curves for each cell, as in fig. 5, is evaluated and divided by the appropriate cell width. The cells at the calorimeter boundary need a special procedure to take into account the unobserved tail, and the cross-talk between cells is also corrected for. The accuracy of this method is about $0.2 \%$. In the "amplitude method" the signal of each individual tile is fitted, taking into account the tails, and it yields the signal amplitude for every tile in the calorimeter. The accuracy of a single tile response is better than $2 \%$ and for the average amplitude of a cell better than $0.3 \%$. The integral method is faster so it is used for a fast equalization of the cells responses (by adjusting the PMTs gains). The amplitude method is used for detailed scans to check the module quality.

In the procedure to equalize the response of every calorimeter cell, the high voltage 


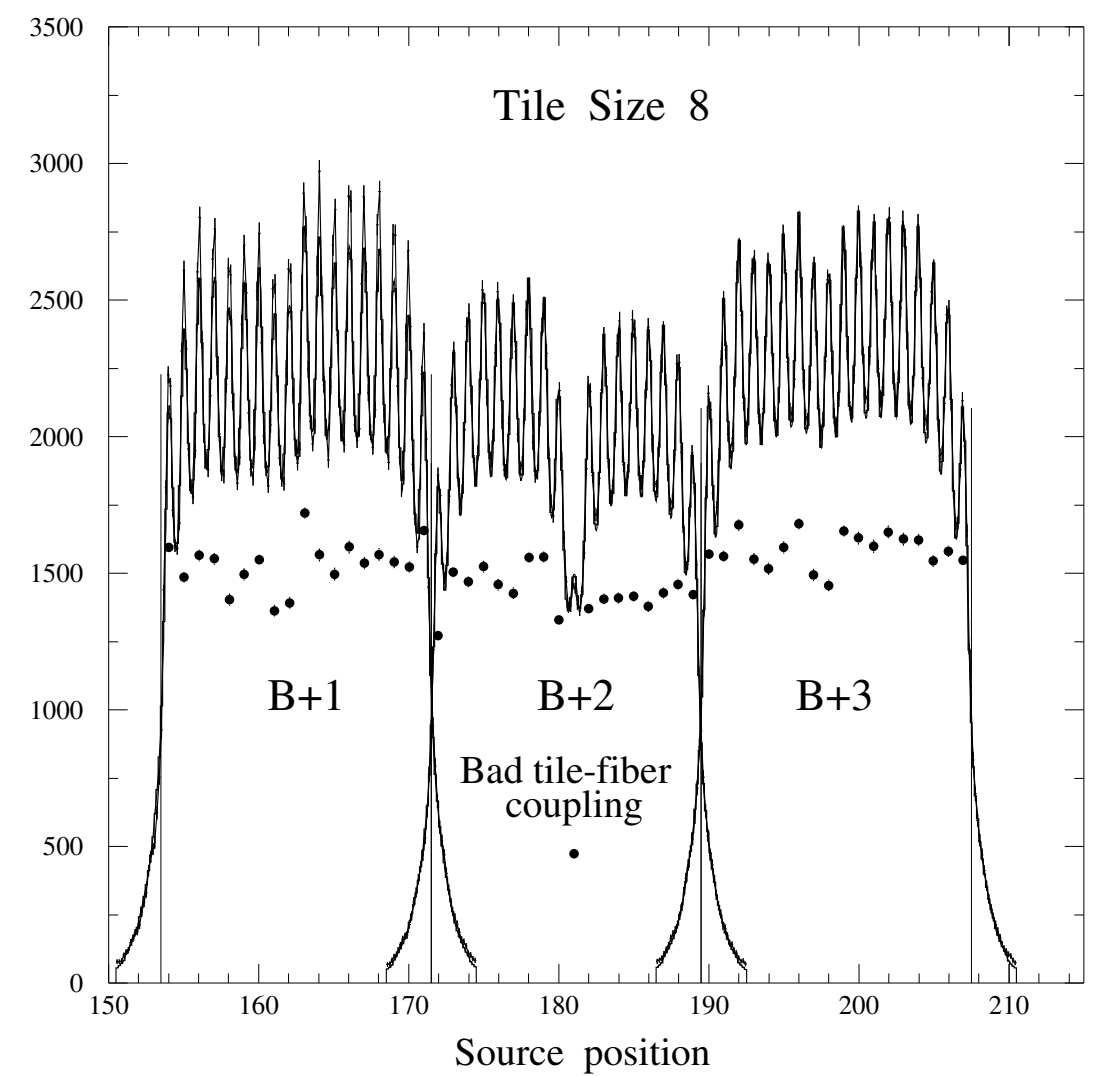

FIGURE 5. The PMT current as a function of the source position for three adjacent cells.

(HV) of each PMT is adjusted in a iterative process to reach a target value of the gain. The new HV values are calculated with the formula:

$$
H V_{\text {new }}=H V_{\text {old }}\left(\frac{\text { target response }}{\text { measured response }}\right)^{1 / \beta}
$$

The parameter $\beta$ changes slightly for each PMT, but a single value, $\beta=7$, gives a convergence to within $0.5 \mathrm{~V}$ in the $\mathrm{HV}$ after three iterations. The target response, the ratio of the measured PMT charge to the deposited electromagnetic energy, was established to be in the range 1.04-1.1 pC/GeV, depending on the angle of the incident particle.

In testbeam the overall cell-to-cell non-uniformity of the calorimeter after the Cesium equalization, as seen by muons and electrons, is less than 3\%. It is worse than the Cesium measurements because the muon and electron beams and the Cesium source deposit energy in different parts of the cells, and the scintillating tiles are not identical (a 5-8\% variation was observed during instrumentation). A hadronic shower spans over many tiles and the response non-uniformity for single pions is at the level of $1.3 \%$.

In ATLAS the Cesium system will be used to monitor the long term stability of the calorimeter. It allows not only to monitor the PMTs stability but also to detect bad tile- 
fiber couplings, scintillator aging and optical problems in general. Between the Cesium runs the PMTs stability is monitored by the laser system.

\subsection{Minimum bias events}

The minimum bias (MB) events in ATLAS are inelastic $p p$ collisions with low momentum transfer, whose rate is proportional to the LHC luminosity [6]. Twenty three MB events per bunch crossing are expected in high luminosity runs. These events produce a non-negligible occupancy of the TileCal cells, with rates uniform in the azimuthal angle $\phi$ and moderately dependent on the pseudo-rapidity $\eta$. Since the MB current, averaged over milliseconds, is almost constant and proportional to the interaction rate, it can be used to monitor the calorimeter response, the relative luminosity and the beam quality during physics runs. Dead and hot channels are also identified in MB data.

For each PMT channel the anode output is DC coupled to an integrator circuit based in an operational amplifier. This has a fixed RC time constant of $10 \mathrm{~ms}$ and a gain selectable from six predefined values (as the MB current changes with the position of the cell on the calorimeter, as well as with the luminosity). All integrator outputs, multiplexed to a 12-bit ADC, are readout every $2 \mathrm{~s}$. In ATLAS, in a high luminosity run, are produced about 8 million MB events during the integration time.

The MB monitoring system was tested with pion beams, at different rates, in order to reproduce the conditions expected in ATLAS and check the system's performance and dynamic range. The results are consistent with the expectations, based on the spills time profile, the average energy deposited, the beam sampling and the MB system design.

\section{CONCLUSIONS}

The ATLAS hadronic calorimeter monitoring and calibration systems were presented. The CIS, laser and Cesium allow to calibrate and to monitor the calorimeter response with a $0.5-1 \%$ precision. After HV equalization the overall cell-to-cell non-uniformity, as measured with electron and muon beams, is better than $3 \%$. This non-uniformity for hadronic showers is at the 1-1.5\% level. Other TileCal monitoring systems, like the HV, the low voltage or the cooling systems, were not presented in this paper.

After the testbeam and the commissioning phases, the different calibration and monitoring systems are ready, waiting for the first ATLAS data taking.

\section{REFERENCES}

1. ATLAS/Tile Calorimeter Collaboration "Tile Calorimeter - Technical Design Report" CERN/LHCC 96-42, 1996.

2. ATLAS/Tile Calorimeter Collaboration, "Testbeam Summary Paper", in preparation, 2006.

3. F. Sarri, "Calibration of the ATLAS Tile Calorimeter", proceedings of the "XI International Conference on Calorimetry in High Energy Physics - CALOR2004", published in "Calorimetry in Particle Physics", World Scientific (2005).

4. E. Starchenko et al., "Cesium monitoring system for ATLAS Tile Hadron calorimeter", Nucl. Instr. an Meth. A494 (2002) 381. 
5. N. Shalanda et al., "Radioactive source control and electronics for the ATLAS Tile Calorimeter cesium calibration system", Nucl. Instr. an Meth. A508 (2003) 276.

6. X. Portell et al., "Distributions of Minimum Bias current measurements in TileCal", ATL-TILECAL03-010, CERN, 2003. 